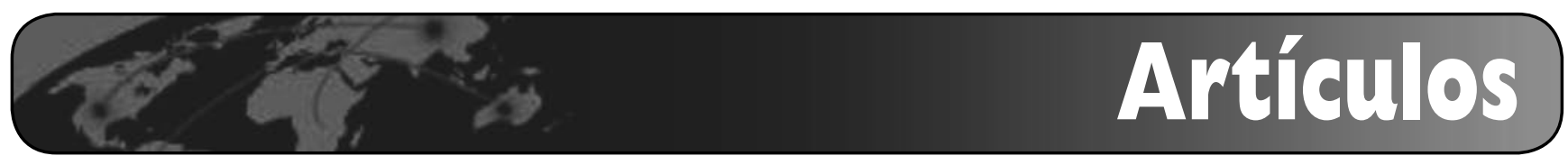

\title{
Edición web distribuida en un portal institucional
}

\author{
Por Rosana López-Carreño
}

\begin{abstract}
Resumen: Se ofrece un estudio de caso en torno a la administración y gestión del contenido de portales institucionales. Se describe la estructura y funcionamiento de un sistema de edición web distribuido, implantado en el portal administrativo de la Consejería de Educación y Cultura de la Región de Murcia, aportándose indicaciones y recomendaciones generales para un óptimo rendimiento del sistema. También se resaltan los beneficios de la descentralización de la edición web como método para consecución de una oferta de contenido de calidad en los portales dependientes de las distintas administraciones públicas, así como la consideración del portal institucional como servicio público añadido a la gestión administrativa.
\end{abstract}

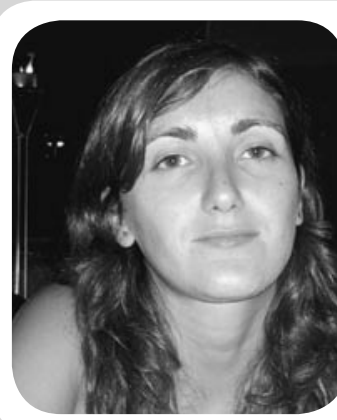

Rosana López-Ca-
rreño, documenta-
lista de la Conse-
jería de Educación
y Cultura de la Re-
gión de Murcia y
profesora asocia-
da de la Facultad
de Comunicación
y Documentación
de la Universidad
de Murcia.

Palabras clave: Edición web distribuida, Portales institucionales, Gestión de contenidos, Auditoría, Administración pública.

\section{Title: Distributed web editing for an institutional portal}

Abstract: This article offers a case study about content administration and management support for institutional portals. It describes the structure and workings of a shared web editing system that has been implemented for the official portal of the Education and Culture Section of Murcia's Regional Government. The article offers general instructions and advice for excellent system performance. It highlights the benefits of decentralized web editing as a method for supplying high quality content in civil service portals and recommends considering an institutional portal to be an added public service in public administration.

Keywords: Shared web editing, Institutional portals, Content management, Audit, Civil service.

López-Carreño, Rosana. "Edición web distribuida en un portal institucional". En: El profesional de la información, 2007, mayo-junio, v. 16, n. 3, pp. 182-187.

DOI: 10.3145/epi.2007.may.02

\section{Introducción}

La puesta en marcha de los portales institucionales se debe fundamentalmente al interés de las administraciones públicas en aprovechar las infraestructuras tecnológicas de la web como marco para incluir una gran variedad de aplicaciones y mejorar la calidad de los contenidos ofertados, sirviendo a públicos potencialmente diferentes y reforzando diversas necesidades informativas.

Entendemos por contenido web del portal el conjunto informativo/documental donde se concentra la información precisa sobre una actividad o trámite administrativo de la organización a la que pertenece, y que está formado por uno o varios archivos electrónicos.

"Los usuarios de los portales institucionales deben tener acceso a información pública de tipo administrativo (portales administrativos) y a información especí- fica de carácter temático (portales temáticos). Por ello, la administración debe fomentar la implementación de ambos tipos de portales y no centrar sus esfuerzos exclusivamente en uno de ellos, puesto que tanto los portales administrativos como los temáticos son de gran interés para el ciudadano" (Martínez; Palacios, 2004). En esta línea, la Consejería de Educación y Cultura de la Región de Murcia llevó a cabo dos sitios paralelos para suplir las distintas necesidades informativas de su amplio espectro de usuarios: uno de carácter temático educativo (Educarm) y otro administrativo o institucional. Dado que "los portales conectan aplicaciones recluidas en silos y distintos repositorios, además de reunir a las audiencias internas y externas" (BEA Syste$m s$ ), el institucional fue objeto de rediseño estructural y conceptual para la mejora del servicio prestado y para la optimización de su gestión. 


\section{http://www.educarm.es}

http://www.carm.es/educacion

Una de las claves de los nuevos diseños de sitios web institucionales es la tendencia a la colaboración en la edición y publicación de contenidos siguiendo las líneas marcadas por la "inteligencia colectiva", potenciada con aplicaciones de código abierto como wikipedias y blogs, ya que "presentan muchas ventajas para editar, dada su flexibilidad en la publicación de modo casi instantáneo de textos e imágenes, democratizando así la producción e interacción de información" (Ferrada, 2006). Esta corriente de trabajo en grupo en la creación y gestión de información por los miembros de una misma administración pública está suponiendo un enriquecimiento de los portales institucionales, advertido positivamente por el ciudadano como un servicio de información fundamental en la sociedad, debido a la actualización casi inmediata, a la precisión informativa y a la especificación documental ofertada.

Siguiendo esta tendencia se estableció una política distribuida en la edición web del contenido del portal institucional, partiendo de la implicación de las distintas unidades directivas y orgánicas de la institución. Esta descentralización implicó un esfuerzo por parte de todas ellas, dado que requería un replanteamiento de la política informativa a seguir por cada una. Además, hubo que establecer perfiles y tareas que concernían al equipo de trabajo interdepartamental que conformaría las nuevas responsabilidades.

Como sucede en cualquier portal, el contenido es considerado la piedra angular de su futuro éxito, siendo su análisis tarea importantísima en este proceso de edición distribuida: "se centra en el estudio de los materiales, servicios e informaciones que son colocados en la sede web" (Rodríguez, 2004). Se realiza una revisión periódica de cada unidad dependiente de la institución, enfocada a suplir la deficiencias informativas-documentales surgidas en dichas unidades, no sólo de las presentes en el portal.

La idea de colaboración está siendo promovida por muchas administraciones públicas que, en una primera fase, pretenden mejorar la actualización y precisión de la información ofrecida a través de la libertad responsable de la producción de contenido y publicación directa en el portal institucional por parte de su personal autorizado (administrador/editor) y cuyo número es susceptible de ampliación en función de futuras necesidades informativas.

\section{Estructura organizativa del sistema de edición web distribuido}

El sistema adoptado permite a cada unidad directi$\mathrm{va}^{1}$ crear y mantener fácilmente sus propios contenidos de forma homogénea y rápida. Cuando el material es de tipo generalista, es decir, cuando su interés es extensible a cualquier tipo de usuario, debe ser analizado y supervisado para ser accesible desde la página principal. Cuando es departamental o específico, se debe publicar en el espacio web de la unidad correspondiente.

Para asegurar la calidad se ha creado una estructura jerarquizada que permite un control y seguimiento de la edición web distribuida, tal y como se indica en la figura 1.

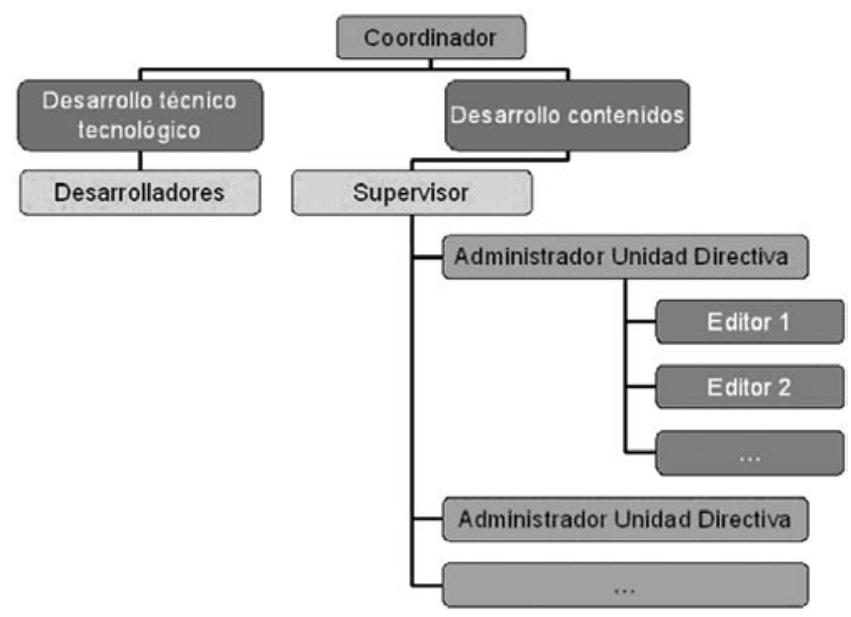

Figura 1. Estructura organizativa

Existen dos áreas claramente diferenciadas: la de desarrollo tecnológico, que se ocupa de la implantación de nuevas aplicaciones y del mantenimiento de las infraestructuras subyacentes en el sistema; y la de contenidos, donde se estructuran, definen y crean los materiales difundidos por el portal institucional.

La estructura de esta última está compuesta por los siguientes responsables:

- Coordinador: establece la política de diseño general del portal y decide la idoneidad de las informaciones de la página principal.

- Supervisor: marca la política de contenidos, los evalúa periódicamente y asesora a los administradores en la creación de nuevos servicios y/o aplicaciones.

- Administradores de unidades directivas: coordinan y representan a los editores dependientes y mantienen los contenidos departamentales de la web de cada unidad. Adicionalmente asesoran sobre la posible inclusión de nuevos materiales.

- Editores: publican directamente contenidos departamentales o específicos.

Con esta estructura se agiliza la disponibilidad de recursos pertinentes en un plazo breve de tiempo. Además, el número de editores está abierto y puede au- 
mentar en función de las necesidades, sin perjuicio de esperar autorización; sólo se solicita a través del administrador de la unidad directiva correspondiente y éste notifica al supervisor el alta del nuevo editor, definiendo sus perfiles y permisos de edición.

La selección del personal que asumirá las responsabilidades de administración y edición web se realiza en función de los siguientes criterios:

- Documentalistas de plantilla de la Consejería.

- Personal funcionario propuesto por cada unidad directiva.

- Personal asesor especializado en materias concretas.

Todos ellos deben poseer conocimientos y destrezas en el desarrollo web, y deben mostrar su interés por el uso del portal institucional como plataforma de agilización de procedimientos y trámites de interés para el usuario potencial.

\section{Acceso a la gestión en el portal institucional}

El sistema de edición distribuido se basa en la utilización de la intranet de la Consejería ${ }^{2}$ como plataforma de acceso a la gestión del contenido, además de proporcionar la entrada al resto de aplicaciones de gestión interna del personal (consulta de datos personales, incidencias, etc.). Una vez definido el $\mathrm{rol}^{3}$ de un nuevo administrador o editor, se habilita el perfil correspondiente, lo que supone su acceso a una serie de aplicaciones y a la publicación en un determinado espacio web dentro del portal institucional. Como puede observarse en la figura 2, el nuevo miembro accederá a una serie de gestores comunes que le auxiliarán en el desarrollo de su web.
Cada sitio que forma parte del portal está constituido por una serie de páginas (definidas por plantillas) y cada una de ellas se divide en zonas donde se presenta un determinado contenido en forma de:

- Página html: para su generación se utiliza el sencillo editor de textos en línea: FCKeditor $^{4}$.

- Tablón de anuncios: se pueden crear, modificar o eliminar tantos como precisen los diferentes webs creados.

- Noticias: es posible actualizar y trabajar con tantos grupos de noticias como se necesite.

- Menús: facilitan el acceso.

Por su parte, el gestor de páginas permite estructurar el sitio web y la distribución del contenido por zonas.

\section{Herramientas metodológicas}

Para auxiliar a los administradores y editores en su tarea, se necesitan herramientas que faciliten la generación y el mantenimiento, como son:

- Diagrama de flujo de trabajo: ayuda a subsanar cualquier duda suscitada en la gestión de cualquier web.

- Manual de procedimiento del administradoreditor: pasos a seguir desde que surge una necesidad informativa hasta que se trasforma en un documento del portal.

- Tutoriales: de las herramientas del sistema y del editor de textos en línea.

- Recomendaciones para la definición y edición web: proporcionan ayuda en la homogeneidad de los materiales.

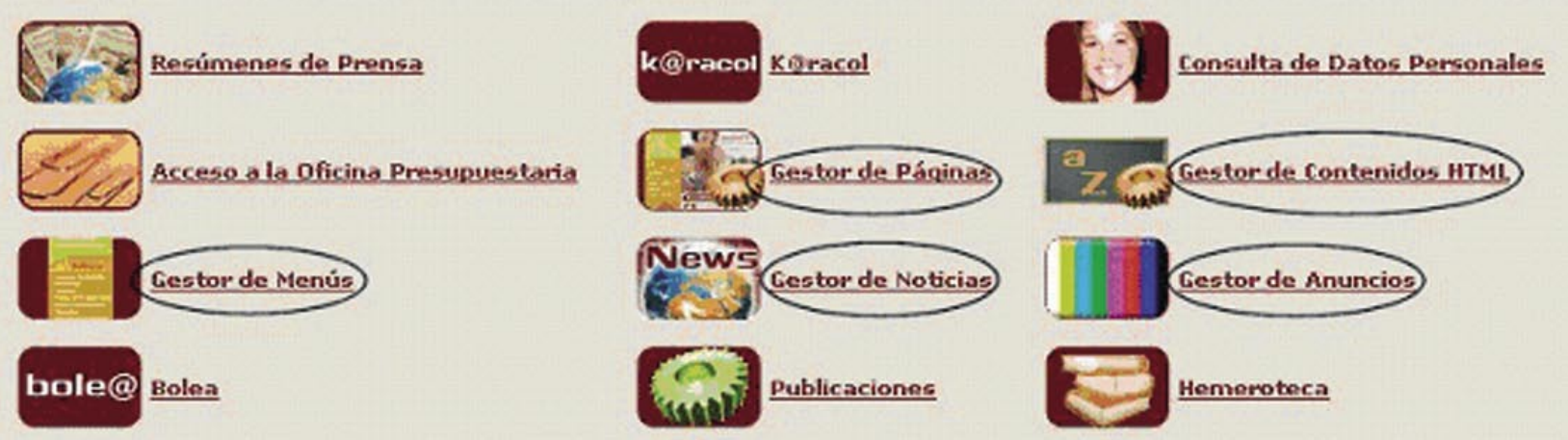


- Indicadores de auditoría: permiten al administrador-editor realizar una primera valoración.

Ante la incorporación de nuevos miembros en la edición web, y para la revisión de la fase de pruebas del contenido generado, es necesario indicar cuáles son los objetivos del mismo:

- Reflejar fielmente la actividad de la institución.

- Facilitar y agilizar la gestión de trámites.

- Solventar necesidades informativas básicas.

\section{Desarrollo del sitio web}

El propósito del sitio es conseguir que el usuario que acceda por primera vez pueda identificar a quién pertenece (unidad directiva y/u orgánica), pueda entenderlo de forma rápida y encontrar fácilmente la información que precisa.

Adicionalmente, un adecuado diseño hará más sencilla la ubicación de nuevas informaciones sin tener que crear nuevas estructuras, y al mismo tiempo conferirá libertad en la incorporación de nuevas aplicaciones en el sitio, sin tener que partir de cero. Para ello es preciso definir una serie de puntos (Rodríguez, 2004) tan relevantes como:

\section{Objetivos del sitio}

Existe uno principal y varios secundarios. "Para generar objetivos que sean válidos y comprensibles, se aconseja escribirlos con frases que se inicien con verbos que expresen las acciones a realizar. Por ejemplo: recibir preguntas de los usuarios sobre las actividades de la unidad directiva, presentar públicamente los informes más importantes de la institución, responder a las preguntas más frecuentes que se reciben en la institución o explicar detalladamente los principales trámites. Permiten cumplir con la idea de tener presencia, a través de una propuesta de contenido que ofrece un valor agregado a quienes ingresan en el sitio" (Gobierno de Chile).

\section{Audiencia}

Definir los principales públicos a los cuales se dirige el portal: usuario interno (personal docente, no-docente...) y externo.

\section{Contenidos del sitio}

Decidir qué materiales se van a ofrecer.

a. Acerca de la unidad directiva: mostrar información completa del organigrama, normativa, oficinas, horas de atención, canales de contacto, etc.

b. Productos/servicios: destacar las actividades principales que el usuario puede hacer en la institución y brindar la información y documentación necesarias para su tramitación. c. Novedades: últimas actividades, noticias, anuncios, etc.

El interés variará si se trata de un usuario interno o externo. "Por ejemplo, si miramos un sitio web desde el punto de vista del usuario externo de la institución, lo que más le interesará será la información sobre trámites, seguida por la que informe de cómo tomar contacto con la institución. Si la miramos desde el punto de vista del usuario interno, lo más importante será la información novedosa, seguida por la de organigrama y normativa" (Rodríguez, 2004).

Es vital la agrupación por categorías o temas, así como un correcto etiquetado del contenido eligiendo las nomenclaturas. Por ello hay que replantearse preguntas tales como: ¿qué significa este nombre? o ¿qué tipos de contenidos esperaría encontrar en esta área?

Con las respuestas obtenidas se podrá juzgar si los nombres que se han usado son los más adecuados o si hay que introducir modificaciones. La búsqueda de otros sitios web similares a nuestra institución, con el fin de revisar de qué manera han resuelto los mismos problemas que deberemos atender, nos puede servir de referencia.

Así mismo es importante una adecuada elección del tipo de información (textual o gráfica) para una acertada interpretación.

Contextualizar la información o documentación que se da al ciudadano es un valor añadido del sitio, ya que facilita el conocimiento de la unidad y del conjunto de la institución. Es erróneo considerar al usuario potencial como si fuera experto; por ello, no hay que dar por hecho que conoce la actividad y trámites que se realizan en la institución. Por ej., hay que evitar simples listas de enlaces externos o internos en pdf.

\section{"Contextualizar la información confiere un valor añadido al sitio ya que facilita el conocimiento de la unidad y del conjunto de la institución"}

\section{Estructura del sitio}

a. Creación de la estructura: identificar qué forma tendrá y tener en cuenta que deberá ser escalable (susceptible de ampliación o modificación). Es preciso distinguir entre estructura y diseño. "Estructura se refiere a la forma que tendrá el sitio en términos generales con sus secciones, funcionalidades y sistemas de navegación. No considera ni incluye elementos gráficos (lo- 
gotipos, viñetas, etc.). Diseño se refiere a la solución gráfica que se creará para el sitio, en la cual aparecen colores, logotipos, viñetas, y otros elementos de diseño que permiten identificar visualmente al sitio" (Rodríguez, 2004).

b. Mapas permanentes: se refiere al proceso de crear un "árbol de contenido" en el que se muestre de manera práctica cuántas secciones estarán en desarrollo y cuántos niveles habrá dentro de cada uno.

b.1. Secciones: se debe intentar que sean las menos posibles, con el fin de concentrar las acciones del usuario en pocas áreas. Se recomienda que el número medio se sitúe alrededor de seis.

b.2. Niveles: hay que intentar que el usuario esté siempre a menos de tres clics del contenido que anda buscando. Por ello es conveniente el uso de los menús para facilitar el acceso a la información. Además, es necesario definir los apartados y el tipo de menú más adecuado a utilizar (cajas, vertical u horizontal).

\section{Diseño visual}

A pesar de quedar limitado este aspecto por las plantillas y hojas de estilo definidas por los desarrolladores tecnológicos, hay que tener presente:

- Imagen corporativa de la institución: se deben seguir los lineamientos de uso de colores, textos y otros elementos definidos.

- Criterios de usabilidad que permitan al usuario entender la estructura del sitio y suplir con satisfacción su necesidad informativa.

- Cuestiones de accesibilidad: que el mayor número posible de personas puedan entrar en la web y usarla, independientemente de las limitaciones propias del individuo o las derivadas del contexto de uso.

\section{Edición del contenido}

Se distinguen dos modalidades de contenido:

- Departamental: elaborado por las distintas unidades orgánicas de la Consejería, de forma individual (por ejemplo, los plazos de un trámite concreto).

- Cooperativo: realizado por las distintas unidades orgánicas de la Consejería colectivamente bajo un mismo soporte (por ejemplo, una base de datos de convocatorias de becas, ayudas y subvenciones).

El primer paso a realizar para su organización conceptual es la formulación de los temas. Para ello es necesario redactar una breve introducción de las principales actividades y trámites llevados a cabo por la institución o unidad administrativa, así como la inclusión de información de contacto y de referencia. Describir de forma clara y exhaustiva el asunto relevante para el usuario, adjuntando la documentación complementaria necesaria en los formatos convenientes también sería útil.

En cuanto a los temas secundarios o vinculados, se debería realizar una breve descripción de los mismos y referenciar o remitir a la unidad administrativa más idónea. Por ejemplo, si una unidad administrativa posee un apartado de las publicaciones monográficas realizadas, deberá referenciar o enlazar al sitio web donde se expongan de forma homogénea todas ellas. Así pues, es necesario el conocimiento de los contenidos de otras unidades administrativas potencialmente relacionadas.

Es importante una revisión periódica de nuestro contenido, por lo que sería conveniente:

- Acceder periódicamente como usuario potencial para observar y anotar las posibles deficiencias informativas percibidas.

- Actualizar la información cuando sea preciso.

- Acceder periódicamente al resto de sitios web del portal y observar aquellos que son susceptibles de vinculación con nuestro sitio web.

- Valorar la idoneidad de desarrollo de nuevas herramientas para la oferta de información de actualización constante.

\section{Indicadores de auditoría}

"Una auditoría de contenido es un repaso conciso de todo el material existente: texto, imágenes, diagramas, etc. Es una excelente oportunidad para determinar qué contenido hay que incorporar al sitio y qué contenido hay que modificar o eliminar" (Goto; Cotler, 2001). En la tabla 1 se indica una serie de indicadores que facilitarán esta tarea y el establecimiento de prioridades en la gestión del sitio.

\section{Conclusiones}

A modo de recopilación final general, podemos apuntar que el reflejo y adopción de las nuevas tendencias en la edición de información en portales institucionales, orientadas a la edición web distribuida, y por tanto hacia la cooperación de los miembros de una misma administración pública, enriquece no sólo el contenido ofertado sino también la imagen proyectada en el usuario potencial y la implicación directa del personal en ese servicio público online, reflejo fiel del servicio público real de la administración.

Por otro lado, las administraciones públicas están impulsando el enriquecimiento de los portales subyacentes de su actividad, convirtiéndolos en valiosos instrumentos en la optimización de la gestión administrativa, mostrando así una apuesta clara del uso de la web 


\begin{tabular}{|c|c|c|c|}
\hline No & Indicador & Sí & No \\
\hline 1 & ¿Están definidos los objetivos de la institución o unidad administrativa? & & \\
\hline 2 & ¿El sitio web representa informativamente a la unidad? & & \\
\hline 3 & ¿Están claramente definidos los trámites de la institución o unidad administrativa? & & \\
\hline 4 & $\begin{array}{l}\text { ¿Es suficiente la información y documentación ofertada para el correcto conocimiento } \\
\text { de un trámite? }\end{array}$ & & \\
\hline 5 & ¿El usuario inexperto puede encontrar fácilmente la información? & & \\
\hline 6 & ¿Es representativa la nomenclatura de los apartados del contenido? & & \\
\hline 7 & ¿Es representativa la nomenclatura del menú propuesto? & & \\
\hline 8 & $\begin{array}{l}\text { ¿Es necesario que el usuario tenga que recurrir al contacto presencial o telefónico para } \\
\text { resolver una duda informativa? }\end{array}$ & & \\
\hline 9 & ¿La estructura del sitio es escalable? & & \\
\hline 10 & $\begin{array}{l}\text { ¿Se accede periódicamente al resto de sitios web del portal y se observa los que son } \\
\text { susceptibles de vinculación con nuestro sitio web? }\end{array}$ & & \\
\hline 11 & ¿Se accede periódicamente a otros sitios web externos similares al nuestro? & & \\
\hline 12 & ¿Se ha planificado la edición de nuevos contenidos? & & \\
\hline
\end{tabular}

Tabla 1. Indicadores de auditoría.

como un eficaz servicio público, añadido a los servicios tradicionales prestados a la comunidad a la que sirven, desplazándose así la idea del web como mero medio de presentación de la institución.

\section{Notas}

1. Se entiende por "unidad directiva" a la secretaría general, direcciones generales y organismos autónomos.

2. Accediendo a través del portal público con un login y password.

3. Los roles permiten controlar los privilegios de cada usuario dentro de la parte privada del portal. Un usuario puede tener uno, varios o ningún rol y un mismo perfil se puede asignar a varios usuarios.

4. Más información en:

http://www.fckeditor.net

\section{Referencias}

BEA Systems, Inc. Estado del sector de los portales en 2006: los portales y los nuevos conocimientos del entorno de la empresa. Consultado en: 0709-06.

http//bea.com
Ferrada, Mariela. "Biblogsfera, comunidad de bitácoras cuya temática versa total o parcialmente sobre biblioteconomía y documentación". En: Biblios, 2006, n. 24

Gobierno de Chile. Guía para el desarrollo de sitios web. Consultado en: 04-10-06.

http://www.guiaweb.gob.cl/index.htm

Goto, Nelly; Cotler, Emily. Rediseño de sitios web. Madrid: Prentice Hall, 2001, ISBN 84-205-3383-1.

Martínez Usero, José Ángel; Palacios Ramos, Elsa. "Los portales científicos como una herramienta para el fomento de la administración electrónica: contenidos y usuarios focalizados". En: Tecnimap, 2004.

Rodríguez Artacho, Miguel, et al. "Propuesta metodológica común para la administración pública para el análisis de proyectos en red pertenecientes a ámbitos educativos, culturales y científicos”. En: Tecnimap, 2004.

Rosana López-Carreño, Facultad de Comunicación y Documentación, Universidad de Murcia, Campus Universitario de Espinardo, 30100 Murcia.

rosanalc@um.es

\section{Versión online de EPI}

\section{Existe una versión electrónica de El profesional de la información,}

de uso gratuito para todos los suscriptores de la revista, que permite acceder a través de internet a los textos completos y materiales gráficos publicados desde el año 2000.

\section{Más información en:}

http://www.elprofesionaldelainformacion.com/contenidos.html 